\title{
On discontinuous implicit differential equations in ordered Banach spaces with discontinuous implicit boundary conditions
}

\author{
by S. CARL (Halle) and S. Heikkil ̈̈ (Oulu)
}

\begin{abstract}
We consider the existence of extremal solutions to second order discontinuous implicit ordinary differential equations with discontinuous implicit boundary conditions in ordered Banach spaces. We also study the dependence of these solutions on the data, and cases when the extremal solutions are obtained as limits of successive approximations. Examples are given to demonstrate the applicability of the method developed in this paper.
\end{abstract}

1. Introduction. Given a real interval $I=\left[t_{0}, t_{1}\right]$ and a Banach space $E$, consider the boundary value problem (BVP)

$$
\left\{\begin{array}{l}
f\left(t, x(t), x^{\prime}(t),-x^{\prime \prime}(t)\right)=0 \quad \text { a.e. on } I \\
C_{i}\left(x\left(t_{0}\right), x\left(t_{1}\right), x^{\prime}\left(t_{0}\right), x^{\prime}\left(t_{1}\right)\right)=0, \quad i=0,1,
\end{array}\right.
$$

where $f: I \times E^{3} \rightarrow E$ and $C_{0}, C_{1}: E^{4} \rightarrow E$. These functions are assumed to satisfy certain monotonicity conditions to be specified later, but they may be discontinuous in all their arguments.

Implicit second order differential equations of the form appearing in (1.1) with nonlinearity $f: I \times \mathbb{R}^{3} \rightarrow \mathbb{R}$ being continuous at least in its last variable, and under standard linear or nonlinear but continuous boundary conditions, have been studied by various authors (cf. e.g. $[4,5,7,9,11,13]$ ). The main approach to the implicit "continuous" differential equations as in (1.1) is to first solve the equation $f(t, x, p, z)=0$ for $z$. However, this approach requires a global continuous solution $z=\widehat{F}(t, x, p)$ to be defined for all $(t, x, p) \in I \times \mathbb{R}^{2}$, which can rarely be obtained.

1991 Mathematics Subject Classification: 34A09, 34G20, 47H07, 47H10.

Key words and phrases: discontinuous implicit differential equations, discontinuous implicit boundary conditions, ordered Banach spaces, fixed point principles in partially ordered sets.

The work on this paper has been supported by the Deutsche Akademische Austauschdienst (DAAD) and by the Finnish Academy. 
A second approach reduces the "continuous" implicit differential equation (1.1) to a differential inclusion of the form

$$
x^{\prime \prime}(t) \in \varphi\left(t, x(t), x^{\prime}(t)\right), \quad t \in I
$$

(cf. e.g. $[5,7,9])$. Existence results for differential inclusion problems have been obtained by various authors (e.g. [2, 3, 4, 9, 10, 12]). However, in all those papers the minimal assumption to treat an inclusion of the form (1.2) is the lower semicontinuity of the multifunction $\varphi$ or at least the existence of a lower semicontinuous multiselection. Moreover, the assumptions on the original nonlinearity $f$ are rather implicit and hard to verify.

Due to the discontinuous nonlinearity $f$ in our implicit differential equation (1.1) none of the existing theories can be applied, even in the case when the functions $f, C_{0}$ and $C_{1}$ are real-valued. In fact, the authors are not aware of any reference dealing with the discontinuous implicit nonlinear boundary value problem of the form (1.1) in Banach spaces. On the other hand, it is well known that continuity of the data does not guarantee the solvability of differential equations in Banach spaces even in the explicit case. In the implicit case regularity conditions alone do not imply the existence of solutions for (1.1).

In this paper we develop a technique that provides existence and comparison results for the implicit boundary value problem (1.1) under explicit and readily verifiable assumptions on the data. This technique is based on the method of upper and lower solutions and a fixed point result in ordered metric spaces, obtained by a generalized iteration method (cf. [6]). Moreover, in some special cases our method allows getting solutions as limits of successive approximations, although the nonlinearities $f, C_{0}$ and $C_{1}$ may be discontinuous in all their arguments. Examples are given to demonstrate applications of the results obtained in this paper.

Throughout this paper we assume that

$E$ is an ordered Banach space with regular order cone $K$, and the partial ordering $\leq$ in $E$, induced by $K$, is defined as $u \leq v$ if and only if $v-u \in K$.

Regularity of $K$ means (cf. [8], p. 36) that each order bounded and monotone sequence of $E$ has a limit.

A function $x$ which belongs to the set

$\mathrm{AC}^{1}(I, E)=\left\{x: I \rightarrow E \mid x^{\prime}\right.$ is absolutely continuous and a.e. differentiable on $I\}$

is called a lower solution of (1.1) if

$$
\left\{\begin{array}{l}
f\left(t, x(t), x^{\prime}(t),-x^{\prime \prime}(t)\right) \leq 0 \quad \text { a.e. on } I, \\
C_{i}\left(x\left(t_{0}\right), x\left(t_{1}\right), x^{\prime}\left(t_{0}\right), x^{\prime}\left(t_{1}\right)\right) \leq 0, \quad i=0,1,
\end{array}\right.
$$

and an upper solution if the reversed inequalities hold. If the equalities hold, we say that $x$ is a solution of (1.1). 
For each function $x \in \operatorname{AC}^{1}(I, E)$ we define $x^{\prime \prime}(t)=0$ at those points $t \in I$ where the second derivative of $x$ does not exist.

We equip the space $\operatorname{AC}^{1}(I, E)$ with the partial ordering $\preceq$ defined by

$$
\begin{aligned}
x \preceq y \text { iff } & x(t) \leq y(t) \text { on } I, x^{\prime}(t) \leq y^{\prime}(t) \text { on } I \text { and } \\
& x^{\prime \prime}(t) \geq y^{\prime \prime}(t) \text { a.e. on } I .
\end{aligned}
$$

If $y, z \in \operatorname{AC}^{1}(I, E)$ and $y \preceq z$, define $[y, z]=\left\{x \in \operatorname{AC}^{1}(I, E) \mid y \preceq x \preceq z\right\}$.

We say that a solution $x_{*}$ of (1.1) is the minimal solution of (1.1) in an order interval $[y, z]$ if $y \preceq x_{*} \preceq x$ for any other solution $x \in[y, z]$ of (1.1), and $x^{*}$ is the maximal solution if $x \preceq x^{*} \preceq z$ for any other solution $x \in[y, z]$ of (1.1). If (1.1) has both the maximal and the minimal solution in $[y, z]$, they are called the extremal solutions of $(1.1)$ in $[y, z]$.

We impose the following hypotheses on (1.1) and on the functions $f$ : $I \times E^{3} \rightarrow E$ and $C_{0}, C_{1}: E^{4} \rightarrow E:$

(f0) There exist a lower solution $y$ and an upper solution $z$ of (1.1) such that $y \preceq z$.

(f1) There is $\mu: I \times E^{3} \rightarrow(0, \infty)$ such that $\mu \cdot f$ is strongly sup-measurable, and the function $w-(\mu \cdot f)(t, u, v, w)$ is nondecreasing in $u, v$ and $w$ for a.e. $t \in I$.

(C) There is $\nu_{i}: E^{4} \rightarrow(0, \infty)$ such that $u_{i}-\left(\nu_{i} \cdot C_{i}\right)\left(u_{0}, v_{0}, v_{1}, u_{1}\right)$ is nondecreasing in all its arguments for $i=0,1$.

Condition (f0) is necessary for the existence of a solution of (1.1), since if $x$ is a solution of (1.1), then (f0) holds when $y=z=x$. In Section 3 we show that conditions (f0), (f1) and (C) are sufficient for the existence of the extremal solutions of $(1.1)$ in the order interval $[y, z]$. Before the proof we derive some auxiliary results.

2. Auxiliaries. We shall first define an operator whose fixed points are solutions of (1.1).

Lemma 2.1. Given $f: I \times E^{3} \rightarrow E, C_{0}, C_{1}: E^{4} \rightarrow E, \mu: I \times E^{3} \rightarrow$ $(0, \infty)$, and $\nu_{0}, \nu_{1}: E^{4} \rightarrow(0, \infty)$, define

$$
\left\{\begin{array}{l}
F x(t)=-x^{\prime \prime}(t)-(\mu \cdot f)\left(t, x(t), x^{\prime}(t),-x^{\prime \prime}(t)\right), \quad t \in I, \\
A_{i} x\left(t_{i}\right)=x^{(i)}\left(t_{i}\right)-\left(\nu_{i} \cdot C_{i}\right)\left(x\left(t_{0}\right), x\left(t_{1}\right), x^{\prime}\left(t_{0}\right), x^{\prime}\left(t_{1}\right)\right), \quad i=0,1
\end{array}\right.
$$

Then $x \in \operatorname{AC}^{1}(I, E)$ is a solution of (1.1) if and only if $F x$ is Bochner integrable on $I$ and $x=G x$, where

$$
\begin{aligned}
G x(t)= & A_{0} x\left(t_{0}\right)+\left(t-t_{0}\right) A_{1} x\left(t_{1}\right) \\
& +\int_{t_{0}}^{t}\left(s-t_{0}\right) F x(s) d s+\left(t-t_{0}\right) \int_{t}^{t_{1}} F x(s) d s .
\end{aligned}
$$


Pr o of. Assume first that $F x$ is Bochner integrable on $I$. From (2.2) it follows by differentiation that

$$
(G x)^{\prime}(t)=A_{1} x\left(t_{1}\right)+\int_{t}^{t_{1}} F x(s) d s
$$

for a.e. $t \in I$. The right-hand side of (2.3) is absolutely continuous and its integral function is $G x$, which implies that (2.3) holds for all $t \in I$. In particular, $(G x)^{\prime}$ is absolutely continuous and a.e. differentiable on $I$. From (2.3) it follows by differentiation that

$$
(G x)^{\prime \prime}(t)=-F x(t) \quad \text { a.e. on } I .
$$

This and (2.1) imply that if $x=G x$, then $x$ satisfies the differential equation of (1.1), and by (2.1)-(2.3) the boundary conditions of (1.1) also hold. Thus $x$ is a solution of (1.1).

Conversely, assume that $x \in \operatorname{AC}^{1}(I, E)$ is a solution of (1.1). Then $x^{\prime}$ is absolutely continuous and a.e. differentiable on $I$, so that $x^{\prime \prime}$ is Bochner integrable on $I$. Because $-x^{\prime \prime}(t)=F x(t)$ a.e. on $I$, it follows that $F x$ is Bochner integrable on $I$. Replacing $F x(s)$ on the right-hand side of (2.2) by $-x^{\prime \prime}(s)$, calculating the integrals obtained and using (2.1) and the boundary conditions of (1.1), one can show that the right-hand side of (2.2) equals $x(t)$ for each $t \in I$. Thus $x=G x$.

Recall that since the order cone $K$ of $E$ is regular, there exists $\gamma>0$ such that

$$
\|u\| \leq \gamma\|v\| \quad \text { whenever } u, v \in K \text { and } u \leq v .
$$

This property is needed in the proof of the following lemma.

Lemma 2.2. Assume that the hypotheses (C), (f0) and (f1) are valid. Then (2.1) and (2.2) define a nondecreasing mapping $G:[y, z] \rightarrow[y, z]$.

Proof. Let $x \in[y, z]$. Conditions (f0) and (f1) imply that the function $F x$ given by (2.1) satisfies

(a) $\quad-y^{\prime \prime}(t) \leq F y(t) \leq F x(t) \leq F z(t) \leq-z^{\prime \prime}(t) \quad$ for a.e. $t \in I$.

From (a) and (2.5) it follows that

$$
\|F x(t)\| \leq(1+\gamma)\left(\left\|y^{\prime \prime}(t)\right\|+\left\|z^{\prime \prime}(t)\right\|\right) \quad \text { for a.e. } t \in I .
$$

This and condition (f1) imply that $F x$ is Bochner integrable. Thus (2.1), (2.2) define a mapping $G x \in \operatorname{AC}^{1}(I, E)$. From the definition of upper and lower solutions it follows by routine calculations that

$$
y \preceq G y \text { and } G z \preceq z .
$$

Assume next that $x, \widehat{x} \in[y, z]$ and $x \preceq \widehat{x}$. By (1.3) this means that

(c) $\quad x(t) \leq \widehat{x}(t)$ and $x^{\prime}(t) \leq \widehat{x}^{\prime}(t)$ on $I$, and $x^{\prime \prime}(t) \geq \widehat{x}^{\prime \prime}(t)$ a.e. on $I$. 
From (2.1) it follows by (c), (f1) and (C) that

$$
\left\{\begin{array}{l}
F x(t) \leq F \widehat{x}(t) \quad \text { for a.e. } t \in I, \\
A_{i} x\left(t_{i}\right) \leq A_{i} \widehat{x}\left(t_{i}\right), \quad i=0,1 .
\end{array}\right.
$$

In view of (2.2)-(2.4) and (d) we have

$$
G x(t) \leq G \widehat{x}(t) \quad \text { and } \quad(G x)^{\prime}(t) \leq(G \widehat{x})^{\prime}(t) \quad \text { on } I,
$$

and

$$
(G x)^{\prime \prime}(t) \geq(\widehat{G} x)^{\prime \prime}(t) \quad \text { a.e. on } I .
$$

This and (1.3) imply that $G x \preceq G \widehat{x}$. Moreover, $G x \in \operatorname{AC}^{1}(I, E)$ for each $x \in[y, z]$.

(Hint: Equations in a proof marked by small letters, e.g. (a), (b), ..., are referred to only in that proof, so that there will be no confusion if one and the same label appears in different proofs.)

Define a norm $\|\cdot\|_{012}$ in $\operatorname{AC}^{1}(I, E)$ by

$$
\|x\|_{012}=\sup _{t \in I}\|x(t)\|+\sup _{t \in I}\left\|x^{\prime}(t)\right\|+\int_{t_{0}}^{t_{1}}\left\|x^{\prime \prime}(t)\right\| d t .
$$

Lemma 2.3. Assume that the hypotheses (C), (f0) and (f1) hold. If $\left(x_{n}\right)_{n=0}^{\infty}$ is a monotone sequence in $[y, z]$, then $\left(G x_{n}\right)_{n=0}^{\infty}$ converges in $[y, z]$ with respect to the norm $\|\cdot\|_{012}$.

Pr o of. Let $\left(x_{n}\right)_{n=0}^{\infty}$ be a nondecreasing sequence in the order interval $[y, z]$ of $\left(\operatorname{AC}^{1}(I, E), \preceq\right)$. By Lemma 2.2 the sequence $\left(G x_{n}\right)_{n=0}^{\infty}$ is nondecreasing and belongs to $[y, z]$. In view of (1.3) this means that the sequences $\left(G x_{n}(t)\right)_{n=0}^{\infty}, t \in I$, are nondecreasing in the order interval $[y(t), z(t)]$ of $(E, \leq)$, the sequences $\left(\left(G x_{n}\right)^{\prime}(t)\right)_{n=0}^{\infty}, t \in I$, are nondecreasing in $\left[y^{\prime}(t), z^{\prime}(t)\right]$ and the sequence $\left(\left(-G x_{n}\right)^{\prime \prime}(t)\right)_{n=0}^{\infty}=\left(F x_{n}(t)\right)_{n=0}^{\infty}$ is nondecreasing in $\left[-y^{\prime \prime}(t),-z^{\prime \prime}(t)\right]$ for a.e. $t \in I$. These results, (2.1) and (C) also imply that the sequences $\left(A_{i} x_{n}\left(t_{i}\right)\right)_{n=0}^{\infty}$ are nondecreasing in $\left[A_{i} y\left(t_{i}\right), A_{i} z\left(t_{i}\right)\right]$ for $i=0,1$. Since the order cone of $E$ is regular, the following limits exist:

$$
\begin{gathered}
x(t)=\lim _{n \rightarrow \infty} G x_{n}(t), \quad v(t)=\lim _{n \rightarrow \infty}\left(G x_{n}\right)^{\prime}(t), \quad t \in I, \\
w(t)=\lim _{n \rightarrow \infty}\left(G x_{n}\right)^{\prime \prime}(t)=\lim _{n \rightarrow \infty}-F x_{n}(t) \quad \text { for a.e. } t \in I, \\
c_{i}=\lim _{n \rightarrow \infty} A_{i} x_{n}\left(t_{i}\right), \quad i=0,1 .
\end{gathered}
$$

Because of (2.2) and (2.3) we have, for each $t \in I$,

$$
\begin{aligned}
G x_{n}(t)= & A_{0} x_{n}\left(t_{0}\right)+\left(t-t_{0}\right) A_{1} x_{n}\left(t_{1}\right) \\
& +\int_{t_{0}}^{t}\left(s-t_{0}\right) F x_{n}(s) d s+\left(t-t_{0}\right) \int_{t}^{t_{1}} F x_{n}(s) d s,
\end{aligned}
$$


and

$$
\left(G x_{n}\right)^{\prime}(t)=A_{1} x_{n}\left(t_{1}\right)+\int_{t}^{t_{1}} F x_{n}(s) d s
$$

Applying the dominated convergence theorem we then obtain $x(t)=c_{0}+\left(t-t_{0}\right) c_{1}+\int_{t_{0}}^{t}\left(s-t_{0}\right)(-w(s)) d s+\left(t-t_{0}\right) \int_{t}^{t_{1}}(-w(s)) d s, \quad t \in I$, and

$$
v(t)=c_{1}+\int_{t}^{t_{1}}(-w(s)) d s, \quad t \in I
$$

This implies that $x \in \mathrm{AC}^{1}(I, E), x^{\prime}(t)=v(t)$ on $I$, and $x^{\prime \prime}(t)=w(t)$ a.e. on $I$. In view of $(\mathrm{d}),(\mathrm{e})$ and $(2.6)$ the sequences $\left(G x_{n}\right)_{n=0}^{\infty}$ and $\left(\left(G x_{n}\right)^{\prime}\right)_{n=0}^{\infty}$ are equicontinuous. From these facts and from (a) it follows that $\left(G x_{n}\right)(t) \rightarrow$ $x(t)$ and $\left(G x_{n}\right)^{\prime}(t) \rightarrow x^{\prime}(t)$ uniformly on $I$. The dominated convergence theorem also implies that

$$
\lim _{n \rightarrow \infty} \int_{t_{0}}^{t_{1}}\left\|\left(G x_{n}\right)^{\prime \prime}(t)-x^{\prime \prime}(t)\right\| d t=0
$$

These results ensure that $G x_{n} \rightarrow x$ with respect to the norm $\|\cdot\|_{012}$, defined by $(2.7)$.

The proof in the case when the sequence $\left(x_{n}\right)_{n=0}^{\infty}$ is nonincreasing is similar.

As a special case of Theorem 1.2.2 of [6] we obtain the following result:

LEMmA 2.4. Let $[\alpha, \beta]$ be a nonempty order interval in $\left(\mathrm{AC}^{1}(I, E), \preceq\right)$, and assume that $G:[\alpha, \beta] \rightarrow[\alpha, \beta]$ is nondecreasing. If $\left(G x_{n}\right)_{n=0}^{\infty}$ converges in $\left(\mathrm{AC}^{1}(I, E),\|\cdot\|_{0,1,2}\right)$ whenever $\left(x_{n}\right)_{n=0}^{\infty}$ is a monotone sequence in $[\alpha, \beta]$, then $G$ has the least fixed point $x_{*}$ and the greatest fixed point $x^{*}$, and

$$
x_{*}=\min \{w \in[\alpha, \beta] \mid G w \preceq w\}, \quad x^{*}=\max \{w \in[\alpha, \beta] \mid w \preceq G w\} .
$$

\section{Existence and comparison results}

3.1. Existence results. Now we are ready to prove our main existence result.

THEOREM 3.1. If conditions $(\mathrm{C}),(\mathrm{f0})$ and $(\mathrm{f} 1)$ are valid, then the BVP (1.1) has the extremal solutions in $[y, z]$.

Pr o of. Assume conditions (C), (f0) and (f1). Lemmas 2.2 and 2.3 ensure that the hypotheses of Lemma 2.4 hold for the operator $G$ defined by (2.1), $(2.2)$, and $[\alpha, \beta]=[y, z]$. Thus $G$ has the least fixed point $x_{*}$ and the greatest fixed point $x^{*}$. In view of Lemma 2.1 this means that $x_{*}$ and $x^{*}$ are the least and the greatest solutions of (1.1) in the order interval $[y, z]$. 
REMARK 3.1. The hypotheses of Theorem 3.1 allow the functions $f, C_{0}$ and $C_{1}$ in (1.1) to be discontinuous with respect to all their arguments.

Consider next the case when certain one-sided continuity hypotheses are satisfied. Given a sequence $\left(u_{n}\right)$ in $E$ converging to $u$, write $u_{n} \nearrow u$ if $\left(u_{n}\right)$ is nondecreasing and $u_{n} \searrow u$ if $\left(u_{n}\right)$ is nonincreasing.

Proposition 3.1. If conditions (f0), (f1) and (C) hold, then the successive approximations

$$
x_{n}=G x_{n-1}, \quad n=1,2, \ldots,
$$

where $G$ is defined by $(2.1),(2.2)$, converge in $\left(\mathrm{AC}^{1}(I),\|\cdot\|_{012}\right)$ to

(a) the minimal solution $x_{*}$ of the $B V P(1.1)$ in $[y, z]$ if $x_{0}=y$ and if $(\mu \cdot f)\left(t, u_{n}, v_{n}, w_{n}\right) \rightarrow(\mu \cdot f)(t, u, v, w)$ for a.e. $t \in I$ and

$$
\left(\nu_{i} \cdot C_{i}\right)\left(s_{n}, u_{n}, v_{n}, w_{n}\right) \rightarrow\left(\nu_{i} \cdot C_{i}\right)(s, u, v, w) \quad \text { for } i=0,1
$$

whenever $s_{n} \nearrow s, u_{n} \nearrow u, v_{n} \nearrow v$ and $w_{n} \nearrow w$;

(b) the maximal solution $x^{*}$ of the BVP (1.1) in $[y, z]$ if $x_{0}=z$ and if $(\mu \cdot f)\left(t, u_{n}, v_{n}, w_{n}\right) \rightarrow(\mu \cdot f)(t, u, v, w)$ for a.e. $t \in I$ and

$$
\left(\nu_{i} \cdot C_{i}\right)\left(s_{n}, u_{n}, v_{n}, w_{n}\right) \rightarrow\left(\nu_{i} \cdot C_{i}\right)(s, u, v, w) \quad \text { for } i=0,1
$$

whenever $s_{n} \searrow s, u_{n} \searrow u, v_{n} \searrow v$ and $w_{n} \searrow w$.

Proof. (a) Choose $x_{0}=y$. From Lemma 2.2 it follows that (3.1) defines a nondecreasing sequence $\left(x_{n}\right)_{n=0}^{\infty}$ in $[y, z]$. Since $x_{n}=G x_{n-1}, n=1,2, \ldots$, by Lemma 2.3 there exists $x_{*} \in[y, z]$ such that $x_{n} \rightarrow x_{*}$ in the norm $\|\cdot\|_{012}$. In particular,

(a) $\quad x_{n}(t) \nearrow x_{*}(t)$ and $x_{n}^{\prime}(t) \nearrow x_{*}^{\prime}(t)$ uniformly on $I$, and

$$
x_{n}^{\prime \prime}(t) \searrow x_{*}^{\prime \prime}(t) \quad \text { a.e. on } I \text {. }
$$

From this and (2.1) it follows by the given continuity hypotheses that

$$
\begin{array}{cl}
\lim _{n \rightarrow \infty} F x_{n}(t)=F x_{*}(t) & \text { for a.e. } t \in I, \\
\lim _{n \rightarrow \infty} A_{i} x_{n}\left(t_{i}\right)=A_{i} x_{*}\left(t_{i}\right) & \text { for } i=0,1 .
\end{array}
$$

These relations, (a), (2.2) and the dominated convergence theorem imply that

$$
x_{*}(t)=\lim _{n \rightarrow \infty} x_{n+1}(t)=\lim _{n \rightarrow \infty} G x_{n}(t)=G x_{*}(t), \quad t \in I .
$$

Thus $x_{*}=G x_{*}$, so that $x_{*}$ is by Lemma 2.1 a solution of (1.1).

If $x$ is any solution of (1.1) in $[y, z]$, we know by Lemma 2.1 that $x$ is a fixed point of $G$, so that $x_{0}=y \preceq x=G x$. Since $G$ is nondecreasing in $[y, z]$ by Lemma 2.2 , it is then easy to see by induction that $x_{n} \preceq x$ for each $n \in \mathbb{N}$, which implies, as $n \rightarrow \infty$, that $x_{*} \preceq x$. This proves that $x_{*}$ is the minimal solution of (1.1) in $[y, z]$.

(b) The proof is similar to case (a). 
3.2. A comparison result. As for the dependence of the extremal solutions of (1.1) on the functions $f, C_{0}$ and $C_{1}$ we have the following result.

Proposition 3.2. If conditions $(\mathrm{C})$, (f0) and (f1) hold, then the extremal solutions of the BVP (1.1) are nonincreasing with respect to the functions $f, C_{0}$ and $C_{1}$.

Pr o of. Assume that conditions (f0), (f1) and (C) are valid for the functions $f, \widehat{f}: I \times E^{3} \rightarrow E$ and $C_{i}, \widehat{C}_{i}: E^{4} \rightarrow E$. Assume also that

(a) $\quad f(t, u, v, w) \geq \widehat{f}(t, u, v, w) \quad$ for a.e. $t \in I$ and all $u, v, w \in E$,

and that

$$
C_{i}\left(u_{0}, v_{0}, v_{1}, u_{1}\right) \geq \widehat{C}_{i}\left(u_{0}, v_{0}, v_{1}, u_{1}\right), \quad u_{i}, v_{i} \in E, i=0,1 .
$$

Thus the hypotheses of Theorem 3.1 also hold when $f, C_{0}$ and $C_{1}$ are replaced by $\widehat{f}, \widehat{C}_{0}$ and $\widehat{C}_{1}$, respectively, so that the boundary value problems (1.1) and

$$
\left\{\begin{array}{l}
\widehat{f}\left(t, x(t), x^{\prime}(t),-x^{\prime \prime}(t)\right)=0 \quad \text { a.e. on } I, \\
\widehat{C}_{i}\left(x\left(t_{0}\right), x\left(t_{1}\right), x^{\prime}\left(t_{0}\right), x^{\prime}\left(t_{1}\right)\right)=0, \quad i=0,1,
\end{array}\right.
$$

have the minimal solutions $x_{*}, \widehat{x}_{*}$ and the maximal solutions $x^{*}, \widehat{x}^{*}$ in the order interval $[y, z]$. In view of Lemma 2.1 we have

$$
\widehat{x}_{*}(t)=\widehat{G} \widehat{x}_{*}(t), \quad t \in I,
$$

where

$$
\begin{aligned}
\widehat{G} x(t)= & \widehat{A}_{0} x\left(t_{0}\right)+\left(t-t_{0}\right) \widehat{A}_{1} x\left(t_{1}\right) \\
& +\int_{t_{0}}^{t}\left(s-t_{0}\right) \widehat{F} x(s) d s+\left(t-t_{0}\right) \int_{t}^{t_{1}} \widehat{F} x(s) d s,
\end{aligned}
$$

with

$$
\left\{\begin{array}{l}
\widehat{F} x(t)=-x^{\prime \prime}(t)-(\mu \cdot \widehat{f})\left(t, x(t), x^{\prime}(t),-x^{\prime \prime}(t)\right), \quad t \in I, \\
\widehat{A}_{i} x\left(t_{i}\right)=x^{(i)}\left(t_{i}\right)-\left(\nu_{i} \cdot \widehat{C}_{i}\right)\left(x\left(t_{0}\right), x\left(t_{1}\right), x^{\prime}\left(t_{0}\right), x^{\prime}\left(t_{1}\right)\right), \quad i=0,1
\end{array}\right.
$$

From (a), (b), (2.1) and (3.4) it follows that

$$
\left\{\begin{array}{l}
F \widehat{x}_{*}(s) \leq \widehat{F} \widehat{x}_{*}(s) \quad \text { for a.e. } s \in I, \\
A_{i} \widehat{x}_{*}\left(t_{i}\right) \leq \widehat{A}_{i} \widehat{x}_{*}\left(t_{i}\right), \quad i=0,1 .
\end{array}\right.
$$

This, (2.2), (3.3), (3.4) and (c) imply that $G \widehat{x}_{*} \preceq \widehat{x}_{*}$. Thus $\widehat{x}_{*} \in[y, z]$ and $G \widehat{x}_{*} \preceq \widehat{x}_{*}$, so that $x_{*} \preceq \widehat{x}_{*}$, by the first formula of $(2.8)$.

Similarly, it can be shown, by applying the second formula of (2.8), that $x^{*} \preceq \widehat{x}^{*}$. 
4. Special cases. In this section we consider

$$
\left\{\begin{array}{l}
-x^{\prime \prime}(t)=g\left(t, x(t), x^{\prime}(t),-x^{\prime \prime}(t)\right) \quad \text { a.e. on } I, \\
x^{(i)}\left(t_{i}\right)=D_{i}\left(x\left(t_{0}\right), x\left(t_{1}\right), x^{\prime}\left(t_{0}\right), x^{\prime}\left(t_{1}\right)\right), \quad i=0,1,
\end{array}\right.
$$

where $g: I \times E^{3} \rightarrow K$ and $D_{0}, D_{1}: E^{4} \rightarrow K, i=0,1$. We make the following assumptions:

(g0) $\quad g$ is a strongly sup-measurable function, and there is $p \in L_{+}^{1}(I)$ such that $g(t, u, v, w)+p(t) w$ is nondecreasing in $u, v$ and $w$ for a.e. $t \in I$.

(g1) $\quad g(t, u, v, w) \leq M(t)(u+v)+h(t)+\lambda(t) w$ for a.e. $t \in I$ and for all $u, v, w \in K$, where $h: I \rightarrow K$ is Bochner integrable, $M /(1-\lambda) \in$ $L_{+}^{1}(I)$, and

$$
\max _{t \in I}\left\{\int_{t_{0}}^{t} \frac{\left(s-t_{0}\right) M(s)}{1-\lambda(s)} d s+\int_{t}^{t_{1}} \frac{\left(t-t_{0}\right) M(s)}{1-\lambda(s)} d s\right\}+\int_{t_{0}}^{t_{1}} \frac{M(s)}{1-\lambda(s)} d s<1 .
$$

(D0) There exist $m_{i} \geq 0$ such that $D_{i}\left(u_{0}, v_{0}, v_{1}, u_{1}\right)+m_{i} u_{i}$ are nondecreasing in $u_{0}, u_{1}, v_{0}$ and $v_{1}$ for $i=0,1$.

(D1) $\quad D_{i}\left(u_{0}, v_{0}, v_{1}, u_{1}\right) \leq d_{i} u_{i}+c_{i}$ for $u_{0}, u_{1}, v_{0}, v_{1} \in K$, where $c_{i} /\left(1-d_{i}\right)$ $\in K, i=0,1$.

LEMma 4.1. Assume that conditions (D1) and (g1) are valid. If $x \in$ $\mathrm{AC}^{1}(I, E)$ is a solution of the $B V P(4.1)$, then $x$ belongs to the order interval $[0, z]$, where $z$ is the solution of the $B V P$

$$
-z^{\prime \prime}(t)=\frac{M(t)\left(z(t)+z^{\prime}(t)\right)+h(t)}{1-\lambda(t)}, \quad z^{(i)}\left(t_{i}\right)=\frac{c_{i}}{1-d_{i}} .
$$

Proof. It is an elementary matter to show (cf. the proof of Lemma 2.1) that $z \in \mathrm{AC}^{1}(I, E)$ is a solution of (4.3) if and only if $z$ is a solution of the equation

$$
z(t)=z_{0}(t)+L z(t), \quad t \in I,
$$

where $L: C^{1}(I, E) \rightarrow C^{1}(I, E)$ and $z_{0} \in C^{1}(I, E)$ are defined by

$$
\begin{aligned}
L x(t)= & \int_{t_{0}}^{t} \frac{\left(s-t_{0}\right) M(s)}{1-\lambda(s)}\left(x(s)+x^{\prime}(s)\right) d s \\
& +\left(t-t_{0}\right) \int_{t}^{t_{1}} \frac{M(s)}{1-\lambda(s)}\left(x(s)+x^{\prime}(s)\right) d s, \\
z_{0}(t)= & \frac{c_{0}}{1-d_{0}}+\frac{\left(t-t_{0}\right) c_{1}}{1-d_{1}} \\
& +\int_{t_{0}}^{t}\left(s-t_{0}\right) \frac{h(s)}{1-\lambda(s)} d s+\left(t-t_{0}\right) \int_{t}^{t_{1}} \frac{h(s)}{1-\lambda(s)} d s, \quad t \in I .
\end{aligned}
$$


Since

$$
(L x)^{\prime}(t)=\int_{t}^{t_{1}} \frac{M(s)\left(x(s)+x^{\prime}(s)\right)}{1-\lambda(s)} d s, \quad t \in I,
$$

condition (4.2) and equations (4.5) and (4.7) imply that, with respect to the norm $\|x\|=\max _{t \in I}\|x(t)\|+\max _{t \in I}\left\|x^{\prime}(t)\right\|$ of $C^{1}(I, E)$, the norm of the linear operator $L$ is less than one. Thus (4.4) and hence also (4.3) has a unique solution $z \in \operatorname{AC}^{1}(I, E)$. This solution and its derivative can be obtained as the limits of the successive approximations

$$
z_{n+1}(t)=L z_{n}(t), \quad z_{n+1}^{\prime}(t)=\left(L z_{n}\right)^{\prime}(t), \quad t \in I .
$$

Since $h$ is $K$-valued and since $c_{i} /\left(1-d_{i}\right) \in K, i=0,1$, it follows from (4.6) that $z_{0}(t) \in K$ and $z_{0}^{\prime}(t) \in K$ for all $t \in I$. But $M /(1-\lambda) \in L_{+}^{1}(I)$, so from (4.5) and (4.7) it follows that $L$ is a positive operator with respect to the partial ordering of $C^{1}(I, E)$ defined by

$$
y \leq z \quad \text { iff } \quad y(t) \leq z(t) \text { and } y^{\prime}(t) \leq z^{\prime}(t) \text { for all } t \in I .
$$

Thus $z(t) \in K$ and $z^{\prime}(t) \in K$ for all $t \in I$. This and (4.3) imply that $-z^{\prime \prime}(t) \in K$ a.e. on $I$. The above proof shows that (4.3) has a unique solution $z \in \operatorname{AC}^{1}(I, E)$, and that $0 \preceq z$.

Assume that $x \in \operatorname{AC}^{1}(I, E)$ is a solution of (4.1). Then $x$ also satisfies the integral equation

$$
\begin{aligned}
x(t)= & x\left(t_{0}\right)+\left(t-t_{0}\right) x^{\prime}\left(t_{1}\right)+\int_{t_{0}}^{t}\left(s-t_{0}\right) g\left(s, x(s), x^{\prime}(s),-x^{\prime \prime}(s)\right) d s \\
& +\left(t-t_{0}\right) \int_{t}^{t_{1}} g\left(s, x(s), x^{\prime}(s),-x^{\prime \prime}(s)\right) d s, \quad t \in I .
\end{aligned}
$$

This implies by differentiation that

$$
x^{\prime}(t)=x^{\prime}\left(t_{1}\right)+\int_{t}^{t_{1}} g\left(s, x(s), x^{\prime}(s),-x^{\prime \prime}(s)\right) d s, \quad t \in I .
$$

Since $g$ and $D_{i}, i=0,1$, are $K$-valued, it follows from (4.1), (4.8) and (4.9) that $x(t) \in K$ and $x^{\prime}(t) \in K$ on $I$, and $-x^{\prime \prime}(t) \in K$ a.e. on $I$. Thus we can apply condition (g1) to show that

$-x^{\prime \prime}(t)=g\left(t, x(t), x^{\prime}(t),-x^{\prime \prime}(t)\right) \leq M(t)\left(x(t)+x^{\prime}(t)\right)+h(t)+\lambda(t)\left(-x^{\prime \prime}(t)\right)$

for a.e. $t \in I$, or equivalently,

$$
\begin{aligned}
-x^{\prime \prime}(t) & =g\left(t, x(t), x^{\prime}(t),-x^{\prime \prime}(t)\right) \\
& \leq \frac{M(t)\left(x(t)+x^{\prime}(t)\right)+h(t)}{1-\lambda(t)} \quad \text { for a.e. } t \in I .
\end{aligned}
$$


From condition (D1) and (4.1) it follows that

$$
x^{(i)}\left(t_{i}\right)=D_{i}\left(x\left(t_{0}\right), x\left(t_{1}\right), x^{\prime}\left(t_{0}\right), x^{\prime}\left(t_{1}\right)\right) \leq d_{i} x^{(i)}\left(t_{i}\right)+c_{i}, \quad i=0,1,
$$

so that

$$
x^{(i)}\left(t_{i}\right) \leq \frac{c_{i}}{1-d_{i}}=z^{(i)}\left(t_{i}\right), \quad i=0,1 .
$$

The integral equations (4.8) and (4.9) and the inequalities (a) and (b) imply that

$$
\begin{aligned}
x(t) \leq & \frac{c_{0}}{1-d_{0}}+\frac{\left(t-t_{0}\right) c_{1}}{1-d_{1}}+\int_{t_{0}}^{t}\left(s-t_{0}\right) \frac{M(s)\left(x(s)+x^{\prime}(s)\right)+h(s)}{1-\lambda(s)} d s \\
& +\left(t-t_{0}\right) \int_{t}^{t_{1}} \frac{M(s)\left(x(s)+x^{\prime}(s)\right)+h(s)}{1-\lambda(s)} d s, \quad t \in I,
\end{aligned}
$$

and

(c) $\quad x^{\prime}(t) \leq \frac{c_{1}}{1-d_{1}}+\int_{t}^{t_{1}} \frac{M(s)\left(x(s)+x^{\prime}(s)\right)+h(s)}{1-\lambda(s)} d s, \quad t \in I$.

In particular, $x$ and $x^{\prime}$ satisfy the inequalities

$$
x(t) \leq z_{0}(t)+L x(t), \quad x^{\prime}(t) \leq z_{0}^{\prime}(t)+(L x)^{\prime}(t), \quad t \in I,
$$

where $L$ and $z_{0}$ are defined by (4.5) and (4.6). On the other hand, $z$ and $z^{\prime}$ are the solutions of the corresponding equalities. Since $L$ is a positive operator, it follows by the Abstract Gronwall Lemma (cf. [14], Prop. 7.15) that $x(t) \leq z(t)$ and $x^{\prime}(t) \leq z^{\prime}(t)$ on $I$. In view of this and (a) we then have

$$
-x^{\prime \prime}(t) \leq \frac{M(t)\left(z(t)+z^{\prime}(t)\right)+h(t)}{1-\lambda(t)}=-z^{\prime \prime}(t) \quad \text { for a.e. } t \in I .
$$

Thus

$$
\left\{\begin{array}{l}
x(t) \leq z(t), \quad x^{\prime}(t) \leq z^{\prime}(t) \quad \text { on } I \\
-x^{\prime \prime}(t) \leq-z^{\prime \prime}(t) \quad \text { for a.e. } t \in I .
\end{array}\right.
$$

From these relations it follows by (1.3) that $x \preceq z$. Moreover, since $g$ and $D_{i}, i=0,1$, are $K$-valued, it follows from (4.1), (4.8) and (4.9) that $0 \preceq x$. Thus $x \in[0, z]$.

The next result is a consequence of Theorem 3.1, Proposition 3.2 and Lemma 4.1.

Proposition 4.1. Assume that conditions (g0), (g1), (D0) and (D1) hold. Then the BVP (4.1) has the extremal solutions $x_{*}$ and $x^{*}$ in the sense that if $x$ is a solution of (4.1), then $x$ belongs to $\left[x_{*}, x^{*}\right]$. Moreover, $x_{*}$ and $x^{*}$ are nondecreasing with respect to $g, D_{0}$ and $D_{1}$. 
Proof. Let

$$
\left\{\begin{array}{l}
f(t, u, v, w)=w-g(t, u, v, w) \\
\mu(t, u, v, w)=\frac{1}{p(t)+1}, \quad t \in I, u, v, w \in E \\
C_{i}\left(u_{0}, v_{0}, v_{1}, u_{1}\right)=u_{i}-D_{i}\left(u_{0}, v_{0}, v_{1}, u_{1}\right), \quad u_{i}, v_{i} \in E, i=0,1 \\
\nu_{i}\left(u_{0}, v_{0}, v_{1}, u_{1}\right)=\frac{1}{m_{i}+1}, \quad u_{i}, v_{i} \in E, i=0,1
\end{array}\right.
$$

Since

$$
w-(\mu \cdot f)(t, u, v, w)=\frac{g(t, u, v, w)+p(t) w}{p(t)+1}, \quad t \in I, u, v, w \in E,
$$

condition (g0) implies that condition (f1) is valid. From

$u_{i}-\left(\nu_{i} \cdot C_{i}\right)\left(u_{0}, v_{0}, v_{1}, u_{1}\right)=\frac{D_{i}\left(u_{0}, v_{0}, v_{1}, u_{1}\right)+m_{i} u_{i}}{1+m_{i}}, \quad u_{i}, v_{i} \in E, i=0,1$,

it follows by condition (D0) that (C) holds. We now show that the solution $z$ of (4.3) and 0 are upper and lower solutions of (1.1) with $f, \mu, C_{i}$ and $\nu_{i}$ given by (4.10). Since $z(t) \in K$ on $I, z^{\prime}(t) \in K$ on $I$ and $-z^{\prime \prime}(t) \in K$ a.e. on $I$, it follows from (4.3), (4.10) and (g1) that

$$
\begin{aligned}
f\left(t, z(t), z^{\prime}(t)\right. & \left.-z^{\prime \prime}(t)\right) \\
& =-z^{\prime \prime}(t)-g\left(t, z(t), z^{\prime}(t),-z^{\prime \prime}(t)\right) \\
& \geq-z^{\prime \prime}(t)-M(t)\left(z(t)+z^{\prime}(t)\right)-h(t)+\lambda(t) z^{\prime \prime}(t) \\
& =(1-\lambda(t))\left(-z^{\prime \prime}(t)-\frac{M(t)\left(z(t)+z^{\prime}(t)\right)+h(t)}{1-\lambda(t)}\right)=0
\end{aligned}
$$

for a.e. $t \in I$. Since $g$ is $K$-valued, we have

$$
f(t, 0,0,0)=0-g(t, 0,0,0) \leq 0, \quad t \in I .
$$

Thus

(a) $\quad f(t, 0,0,0) \leq 0$ and $\quad f\left(t, z(t), z^{\prime}(t),-z^{\prime \prime}(t)\right) \geq 0 \quad$ for a.e. $t \in I$.

In view of (D1), (4.3) and (4.10) we have, for $i=0,1$,

(b) $\quad C_{i}\left(z\left(t_{0}\right), z\left(t_{1}\right), z^{\prime}\left(t_{0}\right), z^{\prime}\left(t_{1}\right)\right)$

$$
=\frac{c_{i}}{1-d_{i}}-D_{i}\left(z\left(t_{0}\right), z\left(t_{1}\right), z^{\prime}\left(t_{0}\right), z^{\prime}\left(t_{1}\right)\right) \geq 0 .
$$

Because $D_{0}$ and $D_{1}$ are $K$-valued, we have

$$
C_{i}(0,0,0,0)=-D_{i}(0,0,0,0) \leq 0, \quad i=0,1 .
$$

From (a)-(c) it follows that 0 is a lower solution and $z$ is an upper solution of (1.1). Moreover, $0 \leq z(t)$ on $I, 0 \leq z^{\prime}(t)$ on $I$ and $0 \leq-z^{\prime \prime}(t)$ a.e. on $I$. Thus condition (f0) holds when $y(t) \equiv 0$ and $z$ is the solution of (4.3). 
The above proof shows that all the hypotheses of Theorem 3.1 are valid, so that (1.1), or equivalently (4.1), has the minimal solution $x_{*}$ and the maximal solution $x^{*}$ in the order interval $[0, z]$. Since all the solutions $x$ of (4.1) belong by Lemma 4.1 to $[0, z]$, it follows that $x_{*}$ is the least and $x^{*}$ is the greatest solution of (4.1).

The last conclusion is a consequence of Proposition 3.2.

Remarks 4.1. (i) We would like to emphasize that the proof of Proposition 4.1 shows that the class of functions $f, \mu, C_{i}$ and $\nu_{i}$ having properties (f0), (f1) and (C) is wide. Namely, examples of such classes of functions are defined by (4.10) combined with conditions (g0), (g1), (D0) and (D1).

(ii) Condition (4.2) can be weakened, for instance by assuming that the operator $T: C^{1}(I, E) \rightarrow C^{1}(I, E)$ defined by

$T x(t)=\int_{t_{0}}^{t} \frac{\left(s-t_{0}\right) M(s)}{1-\lambda(s)}\left(x(s)+x^{\prime}(s)\right) d s+\left(t-t_{0}\right) \int_{t}^{t_{1}} \frac{M(s)}{1-\lambda(s)}\left(x(s)+x^{\prime}(s)\right) d s$ satisfies $\|T\|^{n}<1$ for some $n \in \mathbb{N}$.

If $M(t) /(1-\lambda(t)) \equiv r \geq 0$, then (4.2) reduces to $(r / 2) \cdot\left(\left(t_{1}-t_{0}\right)^{2}+\right.$ $\left.2\left(t_{1}-t_{0}\right)\right)<1$. In particular, if $E=\mathbb{R}$, one can prove the following result:

Proposition 4.2. Let $g: I \times \mathbb{R}^{3} \rightarrow \mathbb{R}$ and $D_{i}: \mathbb{R}^{4} \rightarrow \mathbb{R}, i=0,1$, satisfy the conditions

(g2) $\quad g$ is a Shragin function (cf. [1]) and there is $p \in L_{+}^{1}(I)$ such that $g(t, u, v, w)+p(t) w$ is nondecreasing in $u, v$ and $w$ for a.e. $t \in I$;

(g3) $\quad|g(t, u, v, w)| \leq h(t)+M(t)(|u|+|v|)+\lambda(t)|w|$ for all $u, v, w \in \mathbb{R}$ and for a.e. $t \in I$, where $h, M, \lambda \in L_{+}^{1}(I)$ and

$$
\left\|\frac{M}{1-\lambda}\right\|_{\infty}<\frac{2}{\left(t_{1}-t_{0}\right)^{2}+2\left(t_{1}-t_{0}\right)} ;
$$

(D2) there exists $m_{i} \geq 0$ such that $D_{i}\left(u_{0}, v_{0}, v_{1}, u_{1}\right)+m_{i} u_{i}$ is nondecreasing in $u_{0}, u_{1}, v_{0}$ and $v_{1}$ for $i=0,1$;

(D3) $\left|D_{i}\left(u_{0}, v_{0}, v_{1}, u_{1}\right)\right| \leq d_{i}\left|u_{i}\right|+c_{i}$ for all $u_{0}, u_{1}, v_{0}, v_{1} \in \mathbb{R}$, where $c_{i} /\left(1-d_{i}\right) \geq 0, i=0,1$.

Then the BVP (4.1) has the extremal solutions $x_{*}$ and $x^{*}$, and all the solutions of (4.1) belong to the order interval $[-z, z]$, where $z$ is the solution of the BVP (4.3).

5. Convergence of successive approximations and examples. The next result is an application of Proposition 3.1 to the special case considered in Section 4. We use the following extra conditions: 
(A) $g\left(t, u_{n}, v_{n}, w_{n}\right) \rightarrow g(t, u, v, w)$ for a.e. $t \in I$ and $D_{i}\left(s_{n}, u_{n}, v_{n}, w_{n}\right) \rightarrow$ $D_{i}(s, u, v, w)$ for $i=0,1$ whenever $s_{n} \nearrow s, u_{n} \nearrow u, v_{n} \nearrow v$ and $w_{n} \nearrow w$.

(B) $g\left(t, u_{n}, v_{n}, w_{n}\right) \rightarrow g(t, u, v, w)$ for a.e. $t \in I$ and $D_{i}\left(s_{n}, u_{n}, v_{n}, w_{n}\right) \rightarrow$ $D_{i}(s, u, v, w)$ for $i=0,1$ whenever $s_{n} \searrow s, u_{n} \searrow u, v_{n} \searrow v$ and $w_{n} \searrow w$.

Proposition 5.1. If conditions (g0), (g1), (D0) and (D1) are valid, then the successive approximations

$$
\begin{aligned}
x_{n+1}(t)= & A_{0} x_{n}\left(t_{0}\right)+\left(t-t_{0}\right) A_{1} x_{n}\left(t_{1}\right) \\
& +\int_{t_{0}}^{t}\left(s-t_{0}\right) F x_{n}(s) d s+\left(t-t_{0}\right) \int_{t}^{t_{1}} F x_{n}(s) d s,
\end{aligned}
$$

with

$$
F x_{n}(t)=\frac{g\left(t, x_{n}(t), x_{n}^{\prime}(t),-x_{n}^{\prime \prime}(t)\right)-p(t) x_{n}^{\prime \prime}(t)}{1+p(t)}, \quad t \in I
$$

and

$$
A_{i} x_{n}\left(t_{i}\right)=\frac{D_{i}\left(x_{n}\left(t_{0}\right), x_{n}\left(t_{1}\right), x_{n}^{\prime}\left(t_{0}\right), x_{n}^{\prime}\left(t_{1}\right)\right)+m_{i} x_{n}^{(i)}\left(t_{i}\right)}{1+m_{i}}, \quad i \in 0,1
$$

converge in $\mathrm{AC}^{1}(I, E)$, with respect to the norm $\|\cdot\|_{012}$ defined by (2.7), to

(a) the minimal solution $x_{*}$ of the BVP (4.1) if $x_{0}=0$ and if (A) holds;

(b) the maximal solution $x^{*}$ of the $B V P(4.1)$ if $x_{0}=z$, where $z$ is the solution of (4.3), and if (B) holds.

Proof. (a) The hypotheses of Proposition 3.1 hold when $y=0$ and when $f, \mu, C_{i}$ and $\nu_{i}, i=0,1$, are given by (4.10). Then the successive approximations (5.1) converge to the minimal solution of $(4.1)$ in $[0, z]$. If $x$ is any solution of (4.1), then $x \in[0, z]$, by Lemma 4.1. In view of the choices (4.10) this proves (a).

(b) The proof is similar.

REMARKS 5.1. If the assumptions that $g$ and $D_{i}$ are $K$-valued are replaced by the assumptions that $g(t, 0,0,0) \in K$ for a.e. $t \in I$ and that $D_{i}(0,0,0,0) \in K$, then the results of Sections 4 and 5 hold when one restricts attention to the solutions of (4.1) which satisfy $0 \preceq x$.

If $E=\mathbb{R}$ and if the hypotheses (g2), (g3), (D2) and (D3) hold, then the conclusions of Proposition 5.1 are valid when we choose in the case (a) $x_{0}=-z$, where $z$ is the solution of (4.3).

The results derived in Sections $2-5$ are valid when $E$ is

(a) a finite-dimensional ordered Banach space (cf. [6], Prop. 1.3.1),

(b) $l^{p}, 1 \leq p<\infty$, with componentwise ordering (cf. [6], Ex. 5.8.3), 
(c) an ordered Hilbert space with $(x \mid y) \geq 0$ for all $x, y \geq 0$ (cf. [6], Prop. 5.8.2),

(d) an ordered Banach space with uniformly monotone norm (cf. [6], Prop. 5.8.1),

or one of the following function spaces:

(e) $L^{p}(\Omega, Z), 1 \leq p<\infty$, with a.e. pointwise ordering, where $(\Omega, \mathcal{A}, \mu)$ is a measure space and $Z$ is an ordered Banach space with regular order cone (cf. [6], Prop. 5.8.7),

(f) Orlicz space $L_{M}(\Omega)$, with a.e. pointwise ordering, where $\Omega$ is a bounded domain in $\mathbb{R}^{m}$, and $M$ satisfies the $\Delta_{2}$-condition (cf. [8]),

(g) the space $c_{0}$ of real sequences $x=\left(x_{i}\right)_{i=1}^{\infty}$ with $\lim _{i} x_{i}=0$, equipped with the norm $\|x\|=\sup _{i}\left|x_{i}\right|$ and componentwise ordering (cf. [6], Ex. 5.8.1),

(h) the closure $E_{M}(\Omega)$ of $L^{\infty}(\Omega)$ in $L_{M}(\Omega)$, equipped with a.e. pointwise ordering (cf. [8]).

For other examples of ordered Banach spaces with regular order cones, see for instance [6], Ex. 5.8.4-5.8.6, Cor. 5.8.1-5.8.3 and Prop. 5.8.3.

Example 5.1. Define functions $h_{i}: I \rightarrow \mathbb{R}$ and $q_{i}: \mathbb{R} \rightarrow \mathbb{R}, i=1,2, \ldots$, by

$$
h_{i}(t)=\frac{1}{2^{i}} \sum_{m=1}^{i} \sum_{k=1}^{\infty} \frac{2+\left[k^{1 / m} t\right]-k^{1 / m} t}{(k m)^{2}}\left(2+\sin \left(\frac{1}{1+\left[k^{1 / m} t\right]-k^{1 / m} t}\right)\right)
$$

for $t \in I, i=1,2, \ldots$, and

$$
q_{i}(s)=\frac{1}{2^{i}} \sum_{m=1}^{i} \sum_{k=1}^{\infty} \frac{\pi / 2+\arctan \left(\left[k^{1 / m} s\right]\right)}{(k m)^{2}}, \quad s \in \mathbb{R}, i=1,2, \ldots,
$$

where $[v]$ denotes the greatest integer $\leq v$. By choosing $E=l^{1}$, ordered by the cone $l_{+}^{1}$ of those elements of $l^{1}$ with nonnegative coordinates, consider the BVP

$$
\left\{\begin{array}{l}
-x^{\prime \prime}(t)=g\left(t, x(t), x^{\prime}(t),-x^{\prime \prime}(t)\right) \quad \text { a.e. on } I, \\
x\left(t_{0}\right)=c_{0}, \quad x^{\prime}\left(t_{1}\right)=c_{1},
\end{array}\right.
$$

where $c_{0}, c_{1} \in l_{+}^{1}$, the components of $g=\left(g_{1}, g_{2}, \ldots\right)$ being defined by

$$
g_{i}(t, u, v, w)=h_{i}(t)+q_{i}\left(\sum_{j=1}^{i}\left(u_{j}+v_{j}\right)\right)+\frac{\pi / 2+\arctan \left(\left[-w_{i}\right]\right)}{2^{i}}
$$

for $i=1,2, \ldots, t \in I$ and $u=\left(u_{1}, u_{2}, \ldots\right), v=\left(v_{1}, v_{2}, \ldots\right), w=\left(w_{1}, w_{2}, \ldots\right)$ $\in l^{1}$. It is easy to see that conditions (g0), (g1) and also (D0) and (D1) hold when $D_{i}\left(u_{0}, v_{0}, v_{1}, u_{1}\right) \equiv c_{i}, i=0,1$. Thus by Proposition 4.1 the BVP (5.4) has the extremal solutions $x_{*}$ and $x^{*}$. Since the function $v \mapsto[v]$ is 
right-continuous, it follows from Proposition 5.1 that $x^{*}$ is obtained as a limit of successive approximations.

EXAmPLe 5.2. Consider the BVP

$$
\left\{\begin{array}{l}
-x^{\prime \prime}(t)=\frac{\left[x(t)-t^{2}\right]}{2+2\left|\left[x(t)-t^{2}\right]\right|}+\frac{\left[x^{\prime}(t)\right]}{2+2\left|\left[x^{\prime}(t)\right]\right|}+\frac{\left[-x^{\prime \prime}(t)\right]}{2+2\left|\left[-x^{\prime \prime}(t)\right]\right|} \\
x\left(t_{0}\right)=c_{0}, \quad x^{\prime}\left(t_{1}\right)=c_{1} .
\end{array}\right.
$$

From Proposition 4.2 it follows that (5.5) has the extremal solutions. By Proposition 5.1 the maximal solution is obtained as a limit of successive approximations. These approximations can be calculated by computer, and afterwards one can in some special cases even infer the exact maximal solution. For instance, the maximal solution of (5.5) when $I=[0,1], c_{0}=0$, and $c_{1}=3 / 4$, is

$$
x(t)= \begin{cases}\frac{t}{2}, & 0 \leq t<\frac{1}{2}, \\ \frac{t^{2}}{4}+\frac{t}{4}+\frac{1}{15}, & \frac{1}{2} \leq t \leq 1 .\end{cases}
$$

When $I=[0,1], c_{0}=-1$ and $c_{1}=3 / 4$, the maximal solution of $(5.5)$ is

$$
x(t)= \begin{cases}\frac{t^{2}}{4}+\frac{3 t}{16}-1, & 0 \leq t<\frac{1}{4}, \\ \frac{7}{24} t^{2}+\frac{t}{6}+\frac{383}{384}, & \frac{1}{4} \leq t \leq 1 .\end{cases}
$$

In the case when when $I=[0,1]$ and $c_{i}=1, i=0,1$, the maximal solution of $(5.5)$ is

$$
x(t)=\frac{-t^{2}}{4}+\frac{3}{2} t+1, \quad t \in I .
$$

ExAMPLE 5.3. In our last example

$$
\left\{\begin{array}{l}
-x^{\prime \prime}(t)=\frac{\left[x(t)-t^{2}\right]}{2}+\frac{\left[x^{\prime}(t)\right]}{4}+\frac{\left[-x^{\prime \prime}(t)\right]}{2} \text { a.e. on } I, \\
x\left(t_{0}\right)=\frac{x\left(t_{1}\right)}{2+2\left|\left[x\left(t_{1}\right)\right]\right|}, \quad x^{\prime}\left(t_{1}\right)=\frac{4\left[x\left(t_{0}\right)+1\right]}{1+\left|\left[x\left(t_{0}\right)+1\right]\right|},
\end{array}\right.
$$

also the boundary conditions are implicit. In view of Proposition 5.1(b) and Remarks 5.1 the maximal solution of (5.6) can be obtained by a method of successive approximations. When $I=[0,1 / 2]$, it is

$$
x(t)= \begin{cases}-\frac{t^{2}}{2}+\frac{11}{4} t+\frac{13}{32}, & 0 \leq t<\frac{1}{4} \\ -t^{2}+3 t+\frac{3}{8}, & \frac{1}{4} \leq t \leq \frac{1}{2}\end{cases}
$$

In the case when $I=[1 / 2,1]$, the maximal solution of $(5.6)$ is

$$
x(t)=-\frac{t^{2}}{2}+3 t-1, \quad t \in I .
$$


Acknowledgements. The authors are very grateful to the referee whose comments helped to improve the paper.

\section{References}

[1] J. Appell and P. P. Zabrejko, Nonlinear Superposition Operators, Cambridge Univ. Press, Cambridge, 1990.

[2] G. Bartuzel and A. Fryszkowski, Abstract differential inclusions with some applications to partial differential ones, Ann. Polon. Math. 53 (1991), 67-78.

[3] L. H. Erbe and W. Krawcewicz, Nonlinear boundary value problems for differential inclusions $y^{\prime \prime} \in F\left(t, y, y^{\prime}\right)$, ibid. 54 (1991), 195-226.

[4] L. H. Erbe, W. Krawcewicz and T. Kaczyński, Solvability of two-point boundary value problems for systems of nonlinear differential equations of the form $y^{\prime \prime}=$ $g\left(t, y, y^{\prime}, y^{\prime \prime}\right)$, Rocky Mountain J. Math. 20 (1990), 899-907.

[5] M. Frigon and T. Kaczyński, Boundary value problems for systems of implicit differential equations, J. Math. Anal. Appl. 179 (1993), 317-326.

[6] S. Heikkilä and V. Lakshmikantham, Monotone Iterative Techniques for Discontinuous Nonlinear Differential Equations, Marcel Dekker, New York, 1994.

[7] T. Kaczyński, Implicit differential equations which are not solvable for the highest derivative, in: Delay Differential Equations and Dynamical Systems (Claremont, CA, 1990), S. Busenberg and M. Martelli (eds.), Lecture Notes in Math. 1475, Springer, Berlin, 1991, 218-224.

[8] M. A. Krasnosel'skiı̌, Positive Solutions of Operator Equations, Noordhoff, Groningen, 1961.

[9] S. A. Marano, On a boundary value problem for the differential equation $f\left(t, x, x^{\prime}, x^{\prime \prime}\right)=0$, J. Math. Anal. Appl. 182 (1994), 309-319.

[10] —, Implicit elliptic differential equations, Set-Valued Anal. 2 (1994), 545-558.

[11] W. V. Petryshyn, Solvability of various boundary value problems for the equation $x^{\prime \prime}=f\left(t, x, x^{\prime}, x^{\prime \prime}\right)-y$, Pacific J. Math. 122 (1986), 169-195.

[12] B. Ricceri, Applications de théorèmes de semi-continuité inférieure, C. R. Acad. Sci. Paris Sér. I 295 (1982), 75-78.

[13] S. Stanek, On a class of functional boundary value problems for the equation $x^{\prime \prime}=$ $f\left(t, x, x^{\prime}, x^{\prime \prime}, \lambda\right)$, Ann. Polon. Math. 59 (1994), 225-237.

[14] E. Zeidler, Nonlinear Functional Analysis and its Applications. Vol. I: Fixed-Point Theorems, Springer, Berlin, 1985.

Fachbereich Mathematik und Informatik Institut für Analysis Martin-Luther-Universität Halle-Wittenberg 06099 Halle, Germany

E-mail: carl@condor.mathematik.uni-halle.de
Department of Mathematical Sciences University of Oulu 90570 Oulu, Finland E-mail: sheikki@sun3.oulu.fi 